\title{
Phase Plane Behavior of Solitary Waves in Nonlinear Layered Media
}

\section{Randall J. LeVeque ${ }^{1}$ and Darryl H. Yong ${ }^{2}$}

1 Department of Applied Mathematics, University of Washington, Box 352420, Seattle, WA 98195-2420 rjl@amath.washington.edu

2 Department of Applied and Computational Mathematics, California Institute of Technology, 1200 E California Blvd, MC 217-50 Pasadena, CA 91125 dyong@caltech.edu

\section{Introduction}

The one-dimensional elastic wave equations for compressional waves have the form

$$
\begin{array}{r}
\epsilon_{t}(x, t)-u_{x}(x, t)=0 \\
(\rho(x) u(x, t))_{t}-\sigma(\epsilon(x, t), x)_{x}=0
\end{array}
$$

where $\epsilon(x, t)$ is the strain and $u(x, t)$ the velocity. We consider a heterogeneous material with the density specified by $\rho(x)$ and a nonlinear constitutive relation for the stress given by a function $\sigma(\epsilon, x)$ that also varies explicitly with $x$. This is a hyperbolic system of conservation laws with a spatially-varying flux function, $q_{t}+f(q, x)_{x}=0$.

In particular, we consider a periodic layered medium (a laminate) in which $\rho(x)$ and $\sigma(\epsilon, x)$ are given by

$$
(\rho(x), \sigma(\epsilon, x))= \begin{cases}\left(\rho_{A}, \sigma_{A}(\epsilon)\right) & \text { if } j \delta<x<(j+\alpha) \delta \text { for some integer } j \\ \left(\rho_{B}, \sigma_{B}(\epsilon)\right) & \text { otherwise. }\end{cases}
$$

The two layers have widths $\alpha \delta$ and $(1-\alpha) \delta$ respectively. Here we consider the case $\alpha=1 / 2$ and $\delta=1$.

For sufficiently small strains, the response can be modeled by linear constitutive relations

$$
\sigma_{A}(\epsilon)=K_{A} \epsilon, \quad \sigma_{B}(\epsilon)=K_{B} \epsilon,
$$

where the bulk moduli $K_{A}$ and $K_{B}$ of each material are constants. The equations are then equivalent to acoustics equations in the periodic medium, a case discussed for example in [2], [6]. Here we primarily consider nonlinear equations with

$$
\sigma_{A}(\epsilon)=\exp \left(K_{A} \epsilon\right)-1, \quad \sigma_{B}(\epsilon)=\exp \left(K_{B} \epsilon\right)-1 .
$$

A numerical method for solving more general systems of conservation laws with spatially-varying flux functions has been introduced in [1] and applied 
to the nonlinear systems given above in [4] and [5]. See also [3] for more discussion of the numerical methods used.

An interesting phenomenon has been observed from computations of this nonlinear system: the appearance of solitary waves that arise due to the combination of nonlinearity in the equations and the dispersion induced by reflections at the material interfaces. These solitary waves are investigated in our recent paper [5], where multi-scale homogenization is also applied to obtain a set of homogenized equations that do an excellent job of modeling the behavior seen in the layered medium. The layered medium is also related to the Toda lattice, a discrete nonlinear system that has exact soliton solutions.

Here we present some observations on the structure of these waves that arise from viewing the solitary waves in the phase plane. We observe that Riemann invariants of a related autonomous nonlinear system (with appropriately averaged values of $\rho$ and $K$ ) appear to be significant to the study of wave propagation in this layered medium.

\section{Waves in layered media}

In Fig. 1 we show results of a numerical experiment that is discussed in more detail in [5]. Initially $q(x, 0)=0$ and a wave is generated at the left boundary by the boundary conditions

$$
u(0, t)= \begin{cases}0.2(1+\cos (\pi(t-10) / 10)) & \text { if } 0 \leq t \leq 20 \\ 0 & \text { if } t>20\end{cases}
$$

The left edge is pulled outwards for $0<t<20$, generating a strain wave that propagates to the right. Once the wave has been generated, we switch to periodic boundary conditions so that the wave loops through the domain and the long-time behavior can be studied. In the plot at $t=40$ we see that the smooth hump starts to steepen into a shock followed by a rarefaction, but that oscillations begin to appear behind the shock due to the dispersive nature of the layered medium. At later times these oscillations cause the hump to break up into a train of solitary waves.

Fig. 1 shows the stress, which is continuous at the layer interfaces. Fig. 2 shows a close up view of the first two solitary waves at $t=600$ from Fig. 1, and also shows the corresponding strain $\epsilon$. The strain is discontinuous at the interfaces and so we observe that each solitary wave has a width of about ten layers. These waves are called "stegotons" in [5] because of their ridged appearance. It is not clear whether these waves could possibly be solitons in the technical sense, but they do appear to interact in the same manner as classical solitons. Taller stegotons propagate faster than shorter ones, and when two collide they later separate cleanly into stegotons of the original amplitudes with only a shift in phase (see [5]).

These waves are not smooth when viewed as functions of $x$ at a fixed time $t$. However, if we fix a location $x_{0}$ and record $q\left(x_{0}, t\right)$ as a function of 
time, then the solution is seen to be smoothly varying, as seen in Fig. 3. This approach is used in [5] to study the scaling properties of these waves.
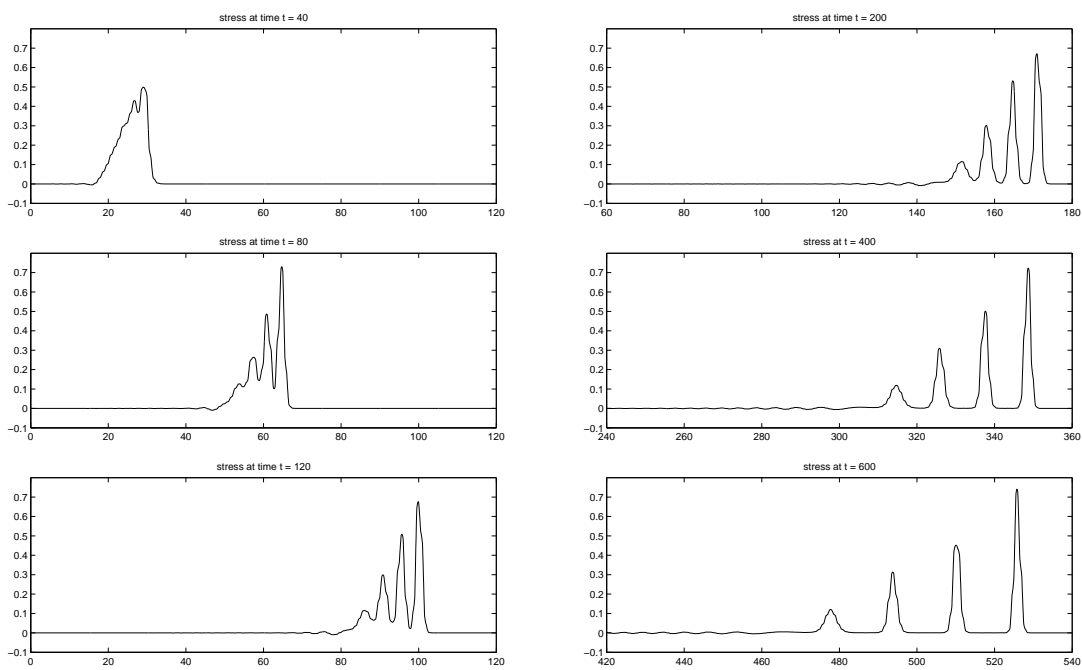

Fig. 1. Propagation in a layered nonlinear medium with impedance mis-match at the interfaces. The left column shows the stress at three times and the right column at three later times, illustrating breakup into a train of solitary waves.
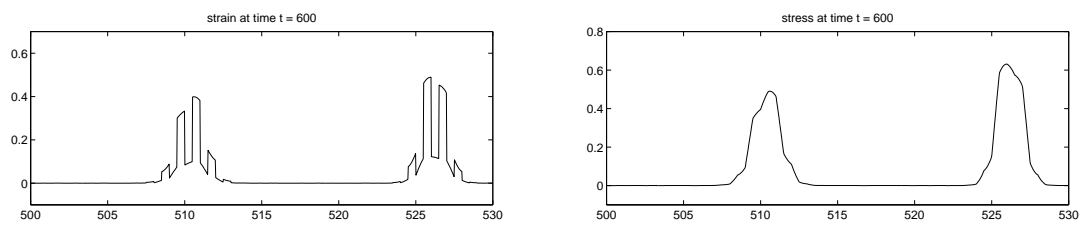

Fig. 2. Close-up view of the first two solitary waves from Fig. 1 at time $t=600$, showing both the strain and the stress.

Here we begin to explore a different view of these waves that we hope may ultimately lead to new insights into their nature. We plot the waves from Fig. 3 in the $\sigma-u$ plane, a variant of the phase plane for this system. What we observe depends on where the point $x_{0}$ lies within the layered structure of the medium. Fig. 4 shows the curves obtained for various locations of $x_{0}$. This computation was performed with 19 grid cells in each layer and the solution was recorded in grid cells 1 through 38 (throughout an A layer and a B layer) over the time period $660 \leq t \leq 700$ as shown in Fig. 3. (Fig. 3 shows the trace for $i=10$, in the middle of the A layer.) The left column of 


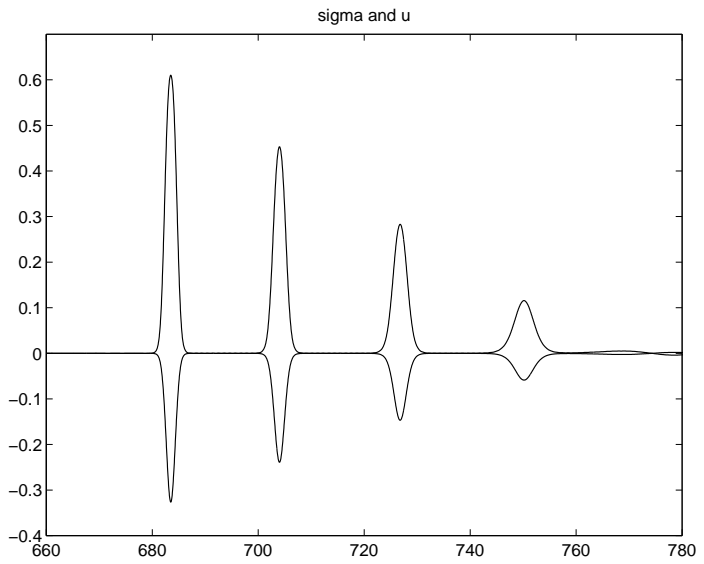

Fig. 3. Time trace of the stress $\sigma\left(x_{0}, t\right) \geq 0$ and velocity $u\left(x_{0}, t\right) \leq 0$ at a fixed point $x_{0}$ as four stegotons pass by.

Fig. 4 shows results at five locations in the A layer, while the right column shows 5 locations in the B layer. In each case four excursions from the origin are observed, corresponding to the four solitary waves.

Note that in the middle of each layer $(i=10$ and $i=19)$, the solitary waves are symmetric in $t$ and the same curve is traced out during the rising and falling portions of the wave. Away from the center of a layer, the excursion traces out a loop, indicating that the wave would not look so symmetric in $t$. In each case, however, the loops appear quite similar for the four stegotons, and scale with the height of the wave.

Note that if we solved the linear equations in a homogeneous medium (i.e., using $\sigma(\epsilon)=K \epsilon$ with $K$ and $\rho$ constant), then a right-going wave would be a simple wave with $u=-\sigma / Z$ everywhere, where $Z=\sqrt{\rho K}$ is the impedance (see [5]). Hence, plots corresponding to Fig. 4 in the homogeneous linear case would show that all values $\left(\sigma\left(x_{0}, t\right), u\left(x_{0}, t\right)\right)$ lie on a line through the origin with slope $-1 / Z$.

For a homogeneous nonlinear material (with $\sigma(\epsilon)=\exp (K \epsilon)-1$ and $K, \rho$ constant), the system (1) becomes

$$
\begin{array}{r}
\epsilon_{t}-u_{x}=0 \\
\rho u_{t}-\exp (K \epsilon)_{x}=0 .
\end{array}
$$

A right-going simple wave would have values $(\sigma, u)$ lying on an integral curve of the eigenvector $r^{2}$ of the Jacobian matrix

$$
f^{\prime}(q)=\left[\begin{array}{cc}
0 & 1 / \rho \\
\sigma^{\prime}(\epsilon) & 0
\end{array}\right]=\left[\begin{array}{cc}
0 & 1 / \rho \\
K e^{K \epsilon} & 0
\end{array}\right] .
$$

The eigenvectors of $f^{\prime}(q)$ are 

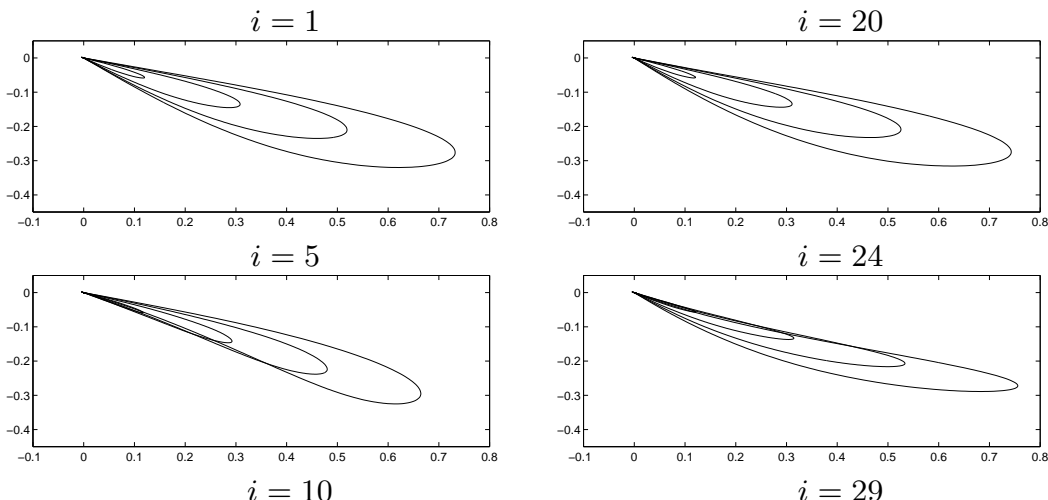

$i=24$
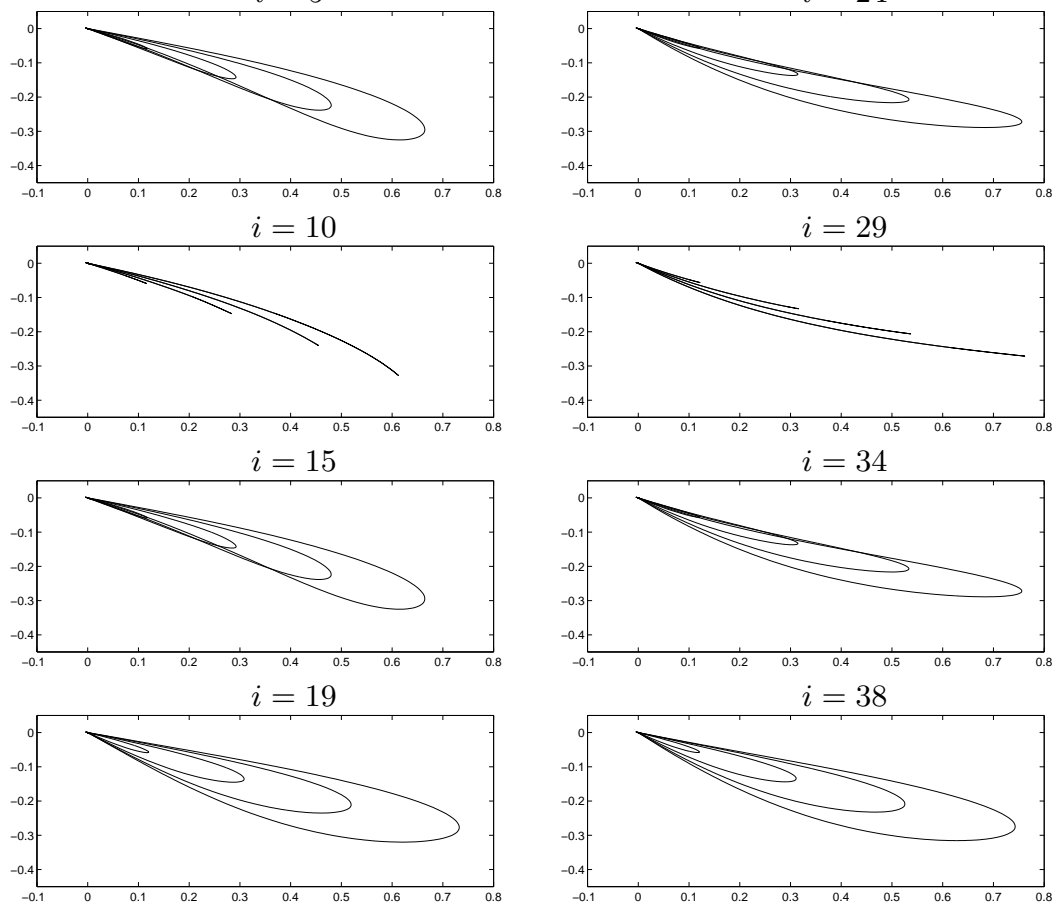

Fig. 4. Solutions of the form shown in Fig. 3 when plotted in the $\sigma-u$ plane. The resulting curves are shown at 5 different locations within each of the two layers (A layer on the left, B layer on the right). The time trace shown in Fig. 3 is at $i=10$, in the middle of an A-layer.

$$
r^{1}=\left[\begin{array}{l}
1 \\
Z
\end{array}\right], \quad r^{2}=\left[\begin{array}{c}
1 \\
-Z
\end{array}\right],
$$

where $Z=\sqrt{\rho \sigma^{\prime}(\epsilon)}=\sqrt{\rho K e^{K \epsilon}}$ varies with $\epsilon(x, t)$ in the nonlinear case.

The Riemann invariants are

$$
w^{1}=\rho u-2 \sqrt{\rho / K} e^{K \epsilon / 2}=\rho\left(u-\frac{2}{\sqrt{\rho K}} \sqrt{\sigma+1}\right)
$$

and

$$
w^{2}=\rho u+2 \sqrt{\rho / K} e^{K \epsilon / 2}=\rho\left(u+\frac{2}{\sqrt{\rho K}} \sqrt{\sigma+1}\right) .
$$


So a right-going simple wave has $w^{2} \equiv$ constant. For a solitary wave that makes an excursion from $u=\epsilon=0$, we must have $w^{2} \equiv 2 \sqrt{\rho / K}$ and so

$$
u=\frac{2}{\sqrt{K \rho}}(1-\sqrt{\sigma+1}) .
$$

Note that $d u /\left.d \sigma\right|_{\sigma=0}=-1 / \sqrt{K \rho}=-1 / Z_{0}$, where $Z_{0}$ is the impedance of the corresponding linearized problem, so this is consistent with the linear case for small amplitude waves.

Homogenization theory (see [5] and [6]) suggests that we might compare the results from the layered medium with integral curves corresponding to a homogenized nonlinear medium with parameters

$$
\bar{\rho}=\frac{1}{2}\left(\rho_{A}+\rho_{B}\right)=5 / 2, \quad \hat{K}=\left(\frac{1}{2 K_{A}}+\frac{1}{2 K_{B}}\right)^{-1}=8 / 5,
$$

and $\sqrt{\bar{\rho} \hat{K}}=2$. There is in fact a close connection. Fig. 5 shows a comparison of two sets of curves from Fig. 4 (for $i=10$ and $i=19$, in the center of each layer) with the integral curve of $r^{2}$ obtained using the values $\bar{\rho}$ and $\hat{K}$ from (11), i.e., the curve $u=1-\sqrt{\sigma+1}$.

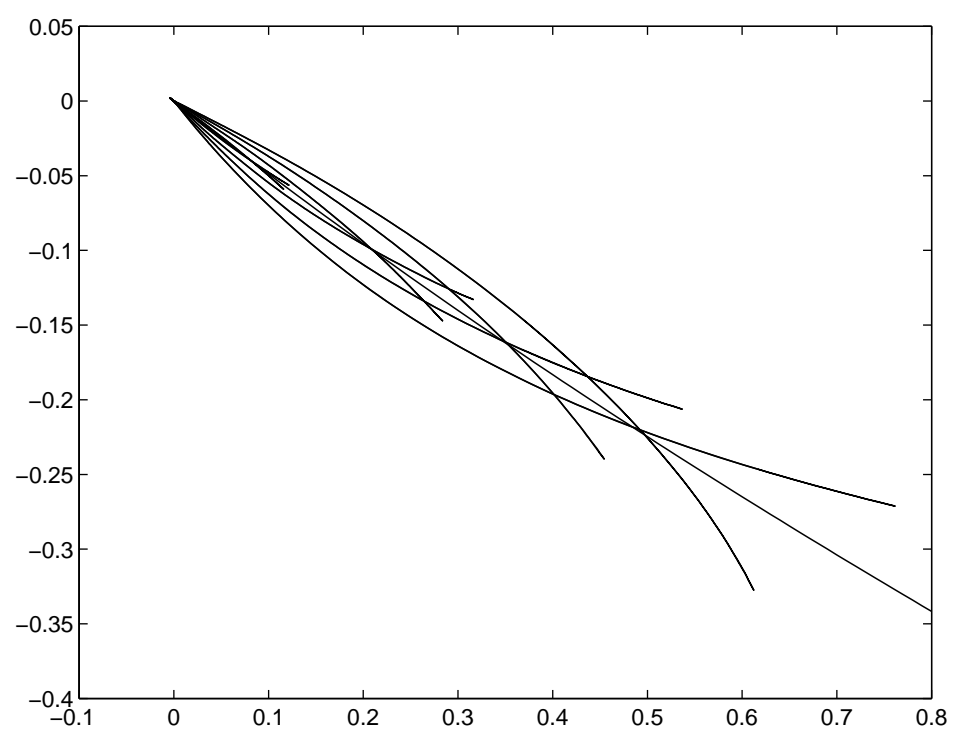

Fig. 5. The curves from Fig. 4 for $i=10$ and $i=29$, together with the integral curve $u=1-\sqrt{\sigma+1}$. 


\section{Homogenization theory}

Nonlinear homogenization theory is developed in [5]. For the case considered here, the following approximate equations are derived:

$$
\begin{aligned}
u_{t}=\frac{2 \sigma_{x}}{5} & +\frac{3 \sigma_{x x x}}{500}+\left(\frac{3 \sigma_{x x x} \sigma_{x}^{2}}{15625(\sigma+1)^{2}}-\frac{72 u_{x x}^{2} \sigma_{x}}{15625(\sigma+1)}-\frac{12 \sigma_{x x x x} \sigma_{x}}{15625(\sigma+1)}\right. \\
& \left.-\frac{96 u_{x x} u_{x x x}}{15625}-\frac{12 \sigma_{x x} \sigma_{x x x}}{15625(\sigma+1)}-\frac{357 \sigma_{x x x x x}}{1000000}\right)+\cdots \\
\sigma_{t}= & \frac{8(\sigma+1) u_{x}}{5}+\left(\frac{3(\sigma+1) u_{x x x}}{125}+\frac{3 u_{x x} \sigma_{x}}{50}\right) \\
& +\left(\frac{48 u_{x} u_{x x}^{2}}{15625}-\frac{48 \sigma_{x} \sigma_{x x} u_{x x}}{15625(\sigma+1)}-\frac{4761 \sigma_{x x x} u_{x x}}{500000}-\frac{72 u_{x x x} \sigma_{x}^{2}}{15625(\sigma+1)}\right. \\
& \left.\quad-\frac{357(\sigma+1) u_{x x x x x}}{250000}-\frac{3543 u_{x x x x} \sigma_{x}}{500000}-\frac{3891 u_{x x x} \sigma_{x x}}{500000}\right)+\cdots
\end{aligned}
$$

These equations have been solved using a pseudospectral method and shown to give results that agree well with solutions to the original layered media equations. Fig. 6 shows solutions to (12) with initial data

$$
u(x, 0)=0, \quad \sigma(x, 0)=\operatorname{sech}^{2}((x-100) / 5) \quad \text { on } \quad 0 \leq x \leq 200,
$$

with periodic boundary conditions. This hump splits up into left-going and right-going pulses that break up into solitary waves in exactly the same manner as observed in the layered medium. At time $t=200$ these wave trains are approaching each other again due to the periodic boundary conditions.
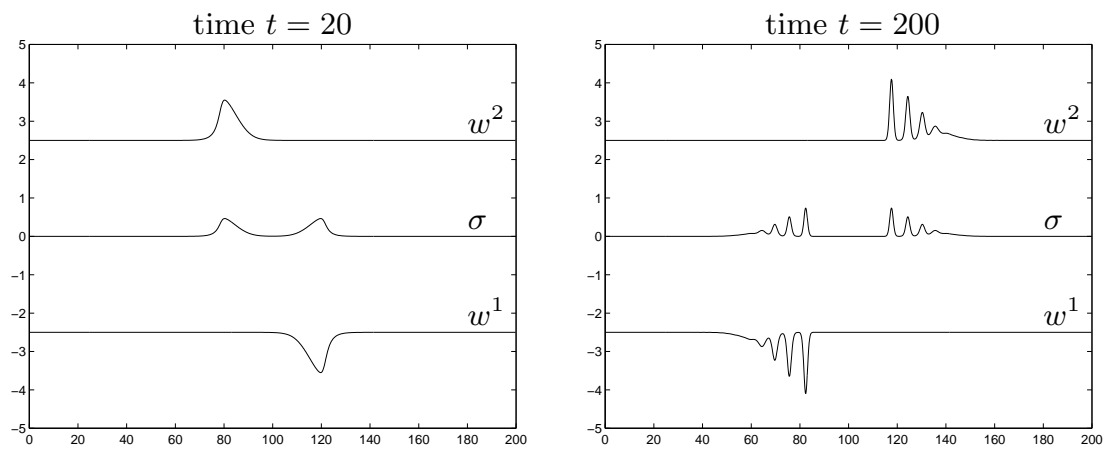

Fig. 6. Solution $\sigma(x, t)$ to the homogenized system of equations (12) at two different times. The Riemann invariants $w^{1}$ and $w^{2}$ corresponding to the first-order hyperbolic system (6) with parameters (11) are computed from this solution and also plotted. 
In these plots, along with $\sigma(x, t)$ we have also plotted the Riemann invariants $w^{1}$ and $w^{2}$ evaluated from the $u$ and $\sigma$ values. Note that the expressions (8) and (9) are based on the simple first-order hyperbolic system for a homogeneous medium (using the parameters (11)), while $u$ and $\sigma$ result from solving the dispersive homogenized equations (12), a system that has very different behavior from the purely hyperbolic system. There is no reason a priori to expect that a left-going train of solitary waves appearing in the solution to (12) will exhibit a constant value of $w^{1}$, but in fact this appears to be true. We see that $w^{1}$ is essentially constant (to about $10^{-3}$ ) through the left-going wave train while $w^{2}$ is essentially constant through the right-going wave train. In Fig. 7 we plot the solution values $(\sigma, u)$ computed from the homogenized equations at $t=200$ together with the integral curves of $r^{1}$ and $r^{2}$, the curves $u= \pm(1-\sqrt{\sigma+1})$. The solution values lie very close to these curves.

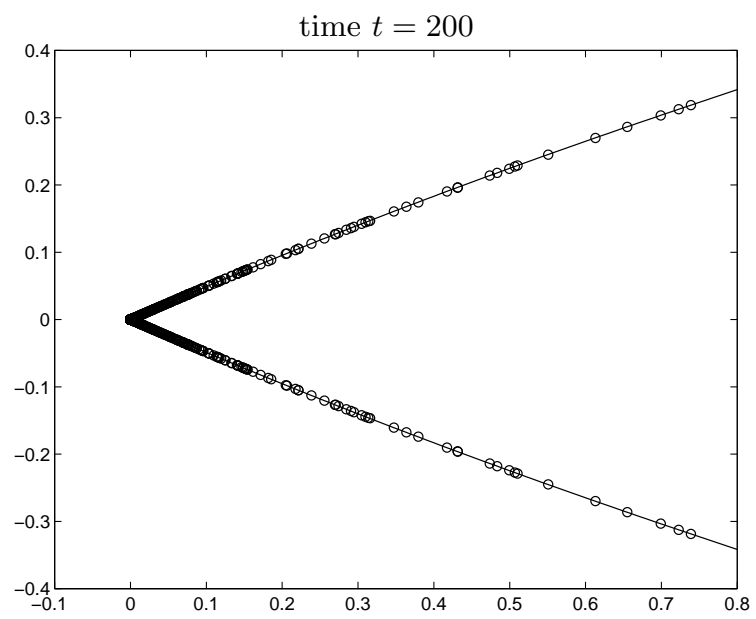

Fig. 7. Solution values from solving the homogenized equations (12), plotted in the $\sigma$ - $u$ plane at time $t=200$. The solid lines are the integral curves $u= \pm(1-\sqrt{\sigma+1})$.

\section{Conclusions}

In the linear case, the constant coefficient system with averaged parameters (11) gives the correct homogenized system to leading order. A pulse propagating in the layered medium is well described by the constant coefficient first-order system for small time, though eventually dispersive effects are visible (see [2], [5], [6]). For the nonlinear problem, however, the first-order nonlinear hyperbolic system (6) with parameters (11) does not adequately 
describe the behavior seen in the layered medium, where the pulse rapidly breaks up into solitary waves. In spite of this, the Riemann invariants of the first order system with averaged parameters appear to be preserved in the wave trains computed using the proper homogenized equations. We are continuing to explore the implications of this observation.

Acknowledgments. This work was supported in part by DOE grant DE-FG03-00ER25292 and NSF grants DMS-9803442 and DMS-0106511.

\section{References}

1. Bale, D., LeVeque, R.J., Mitran, S., Rossmanith, J.R. (2002): A wavepropagation method for conservation laws and balance laws with spatially varying flux functions. SIAM J. Sci. Comput., to appear. (ftp://amath.washington.edu/pub/rjl/papers/fwave.ps.gz).

2. Fogarty, T., LeVeque, R.J. (1999): High-resolution finite volume methods for acoustics in periodic or random media. J. Acoust. Soc. Am. 106, 17-28.

3. LeVeque, R.J. (2002): Finite Volume Methods for Hyperbolic Problems. Cambridge University Press.

4. LeVeque, R.J. (2001): Finite volume methods for nonlinear elasticity in heterogeneous media. Int. J. Numer. Meth. Fluids, to appear. (ftp://amath.washington.edu/pub/rjl/papers/rjl:ox01.ps.gz).

5. LeVeque, R.J., Yong, D.H. (2002): Solitary waves in layered nonlinear media. SIAM J. Appl. Math., to appear. (ftp://amath.washington.edu/pub/rj1/papers/stegoton.ps.gz).

6. Santosa, F., Symes, W. (1991): A dispersive effective medium for wave propagation in periodic composites. SIAM J. Appl. Math. 51, 984-1005. 